\title{
Evaluation of a multispecies probiotic as a supportive treatment for diarrhea in dairy calves: A randomized clinical trial
}

\author{
D. L. Renaud, ${ }^{1 *}$ D. F. Kelton, ${ }^{1}$ J. S. Weese,${ }^{2}$ C. Noble, ${ }^{1}$ and T. F. Duffield ${ }^{1}$ \\ ${ }^{1}$ Department of Population Medicine, University of Guelph, Guelph, ON, Canada N1G 2W1 \\ ${ }^{2}$ Department of Pathobiology, University of Guelph, Guelph, ON, Canada N1G 2W1
}

\section{ABSTRACT}

The objectives of this randomized clinical trial were to determine whether the utilization of a multispecies probiotic bolus (MSP) in dairy calves with diarrhea led to a rapid resolution of diarrhea and improved average daily gain (ADG). Calves, from a convenience sample of dairy farms with diarrhea challenges, having fecal scores of $\geq 2$ were randomly assigned to receive MSP or a placebo (PLB). The MSP bolus contained Pediococcus acidilactici, Enterococcus faecium, Lactobacillus acidophilus, Lactobacillus casei, Bifidobacterium bifidum, peptide extract, an enzyme blend, killed yeast extract, dried whey, and natural flavors (Revive, Partnar Animal Health, Ilderton, ON, Canada). The enrolled calves were fecal scored daily for 7 consecutive days and resolution of diarrhea was defined as having 2 consecutive days with a fecal score $\leq 1$. Calves were also weighed at enrollment, 7 , and $14 \mathrm{~d}$ following enrollment and ADG was calculated. A Cox proportional hazards model was built to investigate time to resolution of an abnormal fecal score. Two mixed linear regression models were created to evaluate the effect of treatment group on ADG in the first and second weeks following enrollment. A total of 148 calves were enrolled in the experiment and no differences were observed between the groups with respect to the age or weight at enrollment. The mean time to resolution of abnormal fecal score was 5.1 and $5.9 \mathrm{~d}$ in the MSP and PLB groups, respectively. In the Cox proportional hazards model, the calves in the MSP group had faster resolution of diarrhea when compared with the PLB group; however, an interaction between time from enrollment of the first calf and treatment group was present. No differences were found between the 2 groups with respect to ADG. This study demonstrates a multispecies probiotic and yeast bolus administered to calves at the onset of diarrhea reduced the duration of diarrhea; however,

Received October 3, 2018.

Accepted January 17, 2019.

*Corresponding author: renaudd@uoguelph.ca the clinical and economic relevance of this reduction requires further exploration.

Key words: diarrhea, probiotic, dairy calves

\section{INTRODUCTION}

Diarrhea is a common cause of morbidity (Svensson et al., 2003; Windeyer et al., 2014; Medrano-Galarza et al., 2018) and mortality (NAHMS, 2007) in young calves, creating significant economic losses to the cattle industry worldwide (Sischo et al., 1990). Thus, it is imperative to employ proper preventative strategies to reduce the incidence of diarrhea as well as provide therapeutics that will mitigate the negative consequences of the disease when it occurs.

The cornerstone of diarrhea treatment is fluid therapy, which is necessary to replace fluid, acid-base, and electrolyte deficits and to provide nutritional support (Smith, 2009). Meloxicam should also be provided to diarrheic calves, which has been shown to result in higher weight gain and increased activity in treated calves (Todd et al., 2010). Antibiotic therapy is suggested only when calves exhibit systemic signs of illness or when blood or mucosal shreds are found in the calf's stool, as antibiotic therapy works to combat bacteremia and bacterial overgrowth in the small intestine (Constable, 2009).

Another potential supportive therapy for diarrheic calves is administration of an oral probiotic and yeast preparations. Probiotics are live microorganisms that, when given at certain doses, exert beneficial health effects. In infants and young children with acute infectious diarrhea, probiotic use has shown some efficacy in reducing the duration of diarrhea (Allen et al., 2010). In calves, research with probiotic species has focused on prevention, with these products fed in the initial portion of the growing period. These experiments have demonstrated that the supplementation of probiotic products can reduce the incidence of diarrhea as well as improve ADG and feed efficiency (Timmerman et al., 2005; Signorini et al., 2012); however, the efficacies of the products vary and are inconsistent (Uyeno et al., 
2015). Currently, no evidence supports using probiotic supplementation as a supportive therapy for diarrhea in calves (Constable, 2009).

We hypothesized that the use of a commercial multispecies probiotic bolus (MSP; Revive, Partnar Animal Health, Ilderton, ON, Canada) would reduce the duration of diarrhea and improve growth following a case of diarrhea. Thus, the objectives of our randomized controlled trial were to determine whether the utilization of a MSP in dairy calves with diarrhea would affect the resolution of diarrhea and ADG.

\section{MATERIALS AND METHODS}

This randomized clinical trial was conducted from May 18 to August 9, 2016, on dairy farms located in southwestern Ontario. The study was approved by the University of Guelph Animal Care Committee (AUP: 3547). Three observers were responsible for enrolling the calves, performing daily fecal scoring, and weighing the calves. The observers, producers, and person responsible for data analysis were blinded to the treatment groups.

\section{Experimental Design}

Farms were selected through a convenience sample of those willing to participate and experiencing diarrhea challenges with their calves. Participating farms housed their calves in group housing on robotic calf feeders or in individual housing in hutches. The maximum quantity of milk or milk replacer provided to calves varied greatly from farm to farm, with a range of 6 to $12 \mathrm{~L}$ of milk being provided per day. The calves were offered milk or milk replacer at a minimum of twice daily to a maximum of ad libitum access. Calves were not enrolled if they had a fecal score of 0 (normal consistency to feces) or 1 (semiformed or pasty feces), whereas calves were enrolled in the study if identified with a fecal score of 2 (runny, spreads easily) or 3 (liquid, devoid of solid material; McGuirk, 2008; Medrano-Galarza et al., 2018). When enrolled, calves were randomly assigned to receive a 4-g bolus of the MSP or a placebo (PLB) orally once daily for 4 consecutive days. Calves were randomized in blocks of 10 according to the RAND command in Microsoft Excel (Version 16.21.1, Microsoft Corp., Redmond, WA). The MSP boluses were from the same batch number and contained dried Pediococcus acidilactici, dried Enterococcus faecium, dried Lactobacillus acidophilus, dried Lactobacillus casei, dried Bifidobacterium bifidum, peptide extract, an enzyme blend, killed yeast extract, dried whey, and natural flavors (Revive, Partnar Animal Health). Before the onset of the trial, the MSP bolus was analyzed by a commercial laboratory (BioSource Flavors Inc., Muskego, WI). It was determined that the total lactic acid bacteria plate count was $43.4 \times 10^{9} \mathrm{cfu} / \mathrm{g}$, with the specific isolation of $41.2 \times 10^{9} \mathrm{cfu} / \mathrm{g}$ of Pediococcus acidilactici, $43.4 \times$ $10^{7} \mathrm{cfu} / \mathrm{g}$ Enterococcus faecium, $43.4 \times 10^{7} \mathrm{cfu} / \mathrm{g} \mathrm{Lac-}$ tobacillus acidophilus, $43.4 \times 10^{7} \mathrm{cfu} / \mathrm{g}$ Lactobacillus casei, and $43.4 \times 10^{7} \mathrm{cfu} / \mathrm{g}$ Bifidobacterium bifidum. The bolus and placebo were found to be negative for coliform species, Escherichia coli, yeast species, Staphylococcus, and Salmonella.

Following enrollment, calves were fecal scored for 7 consecutive days. Calves were weighed using a Dairy Calf Tape (The Coburn Company Inc., Whitewater, WI) at enrollment, 7, and $14 \mathrm{~d}$ following enrollment. Antibiotic treatment and supportive therapy (nonsteroidal anti-inflammatory drugs and intravenous fluids) were recorded for each calf. Calves were excluded if they were $>21 \mathrm{~d}$ of age and had been previously treated with a probiotic supplement.

\section{Sample Size}

The sample size was calculated based on an anticipated time to resolution of diarrhea of $4(\mathrm{SD}=2.5)$ and $5 \mathrm{~d}(\mathrm{SD}=2.5)$ in the MSP and PLB groups, respectively (Katsoulos et al., 2017). Using a $95 \%$ confidence interval and $80 \%$ power, a total of 200 calves or 100 calves per group would be required for this study.

\section{Statistical Analysis}

All statistical analyses were conducted in Stata 14 (StataCorp LP, College Station, TX). Data were imported from Microsoft Excel into Stata 14 and checked for completeness. A causal diagram was created to evaluate the relationships between the potential exploratory variables and the outcomes of interest. Descriptive statistics were generated on all explanatory variables in the data set with $t$-tests and Mann-Whitney tests used to describe continuous normally distributed or non-normal variables, respectively. A $\chi^{2}$ test was used for categorical variables.

To investigate time to resolution of an abnormal fecal score, a Cox proportional hazards model was used. Resolution was defined as having 2 consecutive days with a fecal score $\leq 1$. Kaplan-Meier survival functions were also created to graph time to resolution of abnormal fecal score by group based on the month of enrollment. For the survival functions, a log-rank test was used to determine statistical differences between the groups. Two mixed linear regression models were created to evaluate the effect of treatment on ADG in the first and second weeks following enrollment. In the 3 statistical models, farm was used as a random effect to 
control for the variation between farms. The assumption of linearity of continuous variables in the linear regression models were assessed by plotting outcome against the variable, whereas in the Cox proportional hazards model the functional form of the continuous explanatory variables was assessed by computing the Martingale residuals from a model without the continuous predictor of interest and plotting the residuals against the predictor. If a variable failed to meet the linearity assumption, the variable was categorized. Age and weight at enrollment did not meet the assumption of linearity in any of the models and thus, they were re-categorized based on quartiles. To encompass variation in management practices and pathogen load occurring over time, a variable was created with time from enrollment of the first calf in the experiment being categorized into quartiles. Colinearity among the explanatory variables was tested using Spearman rank coefficients. If the correlation coefficient between 2 variables was $\geq 0.7$, only 1 variable was retained based on fewest missing values, reliability of measurement, or biological plausibility. Univariable regression models were constructed to screen for variables that were unconditionally associated with the outcome using a liberal $P$-value of 0.2 . Risk factors that had univariate associations $(P<0.2)$ were subsequently offered to a multivariable model through a manual backward stepwise process. Evaluating the effect of the removed variables on the coefficients of the remaining variables was used to assess confounding. A variable was deemed to be a confounder if it was not an intervening variable based on the causal diagram and the log odds of a significant variable in the model changed by at least $20 \%$. Two-way interactions were evaluated between treatment and biologically important variables and remained in the final models if significant $(P<0.05$; Dohoo et al., 2010). Homoscedasticity and normality of the residuals and the BLUP were evaluated for model fit in the mixed linear regression models. Outliers were identified and evaluated using standardized residuals as well as Cook's D, DFITS, and DFBETA. In the Cox proportional hazards model, proportionality assumption was tested graphically using log-log plots. If outliers were found in any of the models, they were explored to determine the characteristics of the observations that made them outliers and ensure data were not erroneous.

\section{RESULTS}

\section{Descriptive Results}

Nine dairy farms participated in the project, enrolling 168 Holstein calves. Twenty calves were excluded from the analysis due to loss to follow-up, where the fecal score was not completed for 7 consecutive days (Figure 1). Thus, 68 calves were enrolled in the MSP group and 80 calves were enrolled in the PLB group. Table 1 outlines the number of calves enrolled and the number of calves developing diarrhea on each farm. We found no differences observed between the groups with respect to the age or weight at enrollment (Table 2). Four calves died during the study period $(2.7 \%)$, with no differences observed between the 2 groups (Table 2 ). Treatment (supportive or antibiotic therapy) was administered to $20(13.5 \%)$ and $15(10.1 \%)$ calves in the 1st and 2nd week following enrollment, respectively. No differences were observed between the groups with respect to supportive or antibiotic treatment (Table 1).

\section{Resolution of Diarrhea}

The mean time to resolution of abnormal fecal score was $5.5 \mathrm{~d}$ (Table 1). The calves enrolled in the MSP group had a faster resolution of diarrhea when compared with the PLB group when evaluated with a Wilcoxon rank-sum test (Table 2).

Treatment group, weight, and time from first enrollment in the experiment were unconditionally associated with the outcome and were entered into the mixed multivariable Cox proportional hazards model with farm as a random effect. Only treatment group and time from first enrollment were significant in the final model, with an interaction present between time from first enrollment and treatment group (Table 3). The final model indicated that, at any particular time, the MSP group had 2.4 times as many resolved cases of diarrhea compared with the PLB group. The Kaplan-Meier survival function demonstrated visually a difference in time to resolution of abnormal fecal score when stratified by time from first enrollment (Figure 2). Differences were found between the groups in first quartile $(P=0.01)$ of time following the first enrollment, but not in the second quartile $(P=0.55)$, third quartile $(P=0.35)$, or fourth quartile $(P=0.09)$.

\section{ADG in the First Week Following Enrollment}

The mean ADG in the first week following enrollment was $0.59 \mathrm{~kg} / \mathrm{d}$. The PLB group had a mean of $0.58 \mathrm{~kg} / \mathrm{d}$ of gain compared with the MSP, with a mean of $0.63 \mathrm{~kg} / \mathrm{d}$ of gain $(P=0.60)$. The variables that were unconditionally associated with ADG in the first week following enrollment were age at enrollment and time following enrollment of the first calf. Treatment group was forced into the final model despite not being unconditionally associated. In the multivariable model, only age at enrollment was significant; however, for 


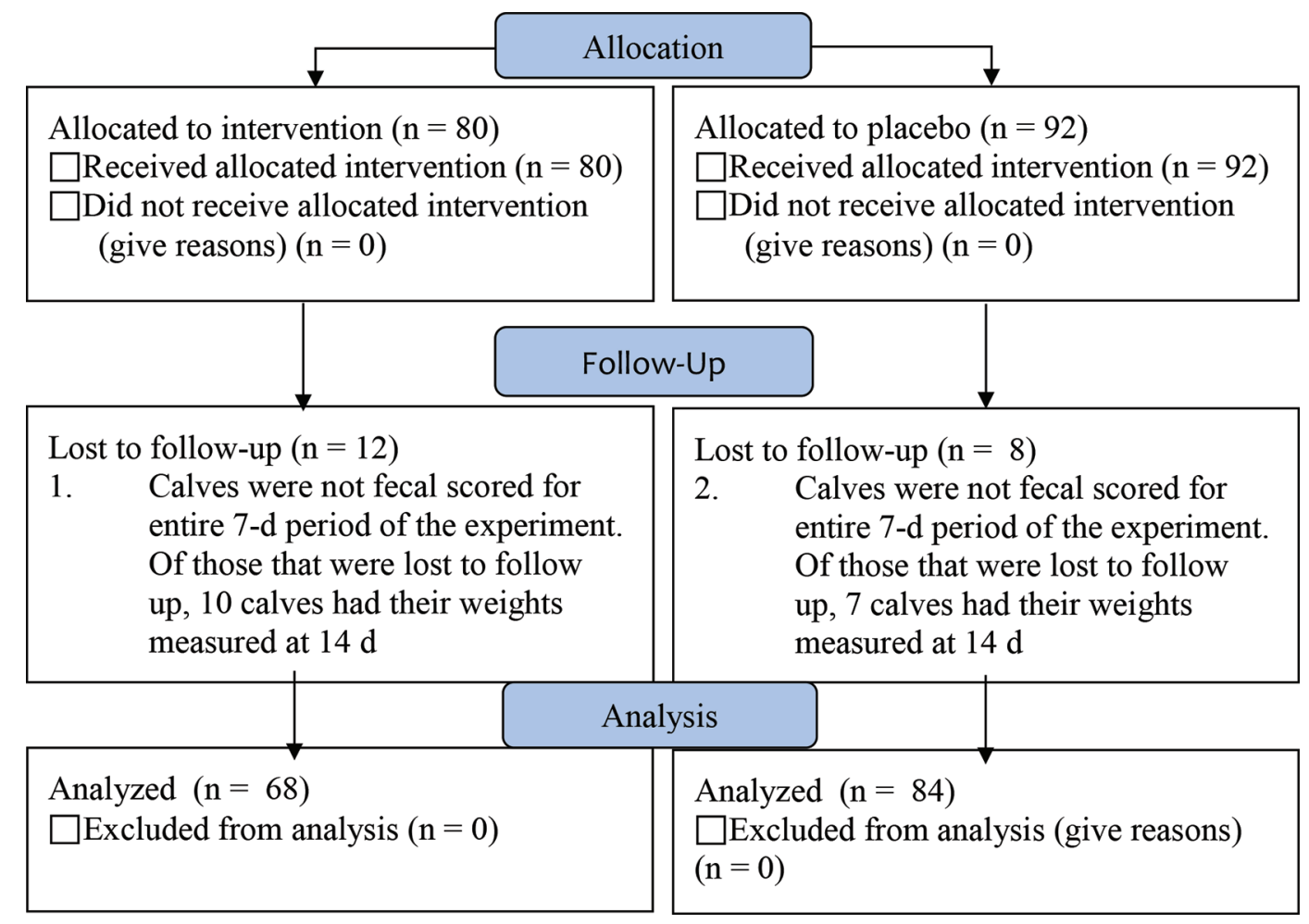

Figure 1. Study flow diagram of the progress through the phases of a randomized trial evaluating the efficacy of a multispecies probiotic bolus in the treatment of diarrhea in dairy calves (CONSORT, 2010).

descriptive purposes, treatment group was kept in the model (Table 4). Time following enrollment was also retained in the model due to a significant $F$-test. We identified no outliers in the model.

\section{ADG in the First 2 Weeks Following Enrollment}

In the first 2 wk following enrollment, the mean $\mathrm{ADG}$ was $0.78 \mathrm{~kg} / \mathrm{d}$. The PLB group had a mean of $0.79 \mathrm{~kg} / \mathrm{d}$

Table 1. Resolution of diarrhea in 148 calves evaluated for 7 consecutive days on 9 farms in southwestern Ontario, Canada, in the multispecies probiotic (MSP) and placebo (PLB) groups

\begin{tabular}{|c|c|c|c|c|c|}
\hline $\begin{array}{l}\text { Farm } \\
\text { number }\end{array}$ & $\begin{array}{l}\text { Proportion of examined calves identified } \\
\text { with diarrhea and enrolled into experiment }\end{array}$ & Group & $\mathrm{N}$ & $\begin{array}{l}\text { Mean resolution } \\
\text { of diarrhea }(\mathrm{d})\end{array}$ & SD \\
\hline \multirow[t]{2}{*}{1} & $40(6 / 15)$ & PLB & 3 & 5.5 & 3.5 \\
\hline & & MSP & 3 & 8.0 & 0 \\
\hline 2 & & MSP & 7 & 3.9 & 1.5 \\
\hline \multirow[t]{2}{*}{3} & $100(8 / 8)$ & PLB & 5 & 6.6 & 2.2 \\
\hline & & MSP & 3 & 3.7 & 1.5 \\
\hline \multirow[t]{2}{*}{5} & $33(5 / 15)$ & PLB & 3 & 8 & 0 \\
\hline & & MSP & 2 & 8 & 0 \\
\hline \multirow[t]{2}{*}{6} & $60(3 / 5)$ & PLB & 1 & 8 & 0 \\
\hline & & MSP & 2 & 6 & 2.6 \\
\hline \multirow[t]{2}{*}{7} & $33(2 / 6)$ & PLB & 1 & 5 & 4 \\
\hline & & MSP & 1 & 0 & 0 \\
\hline 8 & $44(89 / 202)$ & PLB & 46 & 6.2 & 5.2 \\
\hline
\end{tabular}


Table 2. Characteristics of the calves contained in the multispecies probiotic (MSP) group $(\mathrm{n}=68)$ and the placebo (PLB) group $(\mathrm{n}=80)$

\begin{tabular}{|c|c|c|c|}
\hline Variable & MSP & PLB & $P$-value \\
\hline Age at enrollment (d) & 10.6 & 10.6 & 0.95 \\
\hline Mortality [\% (no.)] & $2.9(2)$ & $2.5(2)$ & 0.87 \\
\hline Treatment (supportive or antibiotic therapy) wk 1 and 2 after enrollment [\% (no.)] & $23.5(16)$ & $23.8(19)$ & 0.98 \\
\hline Time until resolution of diarrhea $(\mathrm{d})$ & 5.1 & 5.9 & 0.04 \\
\hline
\end{tabular}

of gain compared with the MSP, with a mean of 0.76 $\mathrm{kg} / \mathrm{d}$ of gain $(P=0.30)$. Time following enrollment of the first calf was the sole variable unconditionally associated with ADG in the first 2 wk following enrollment. In the final model, with treatment group forced into the model, being in the last quartile of time following enrollment resulted in a $0.10 \mathrm{~kg} / \mathrm{d}$ reduction in growth in the 2 wk following enrollment $(P=0.02)$. Treatment group was not significant and no interactions were found. We identified no outliers in the model.

\section{DISCUSSION}

This study demonstrated that the use of an MSP reduced the duration of diarrhea; however, the effect was dependent on the time when the calf was enrolled in the experiment and, with the small effect size, the clinical and economic relevance of this finding requires further exploration. With respect to ADG, we found no difference in the first week following enrollment or the first 2 wk following enrollment when comparing the 2 treatment groups; however, the results could have been strengthened through the use of a weigh scale instead of measuring the stature of the calf with a heart girth weight tape. Some limitations should be considered when interpreting the results of our study. Three observers were responsible for evaluating the calves, and no inter- or intra-observer reliability was performed to compare the results between the observers; however, the observers were trained by a veterinarian using the same scoring rubric to ensure consistency between observ- ers. Loss to follow bias could have occurred with the 20 calves that were lost; however, with similar losses in the 2 groups, this bias may have been minimized (Dohoo et al., 2010). An uneven number of calves were enrolled into each treatment group from the onset of the experiment, with 92 in the PLB group and 80 in the MSP group. The randomized blocks of 10 were heavily weighted in the initial portion of the block with PLB groups and, due to the low number of calves recruited on some farms, more calves were recruited into the PLB groups. Despite the groups being similar with respect to BW and age at enrollment, the inability to control for failure of passive transfer of immunity status and the underlying pathogen could have influenced the results, as they have been shown to influence diarrhea resolution (Goodell et al., 2012). We also failed to achieve the expected sample size due to enrollment only occurring in the summer months when students where available. This could have led to an underpowered study, leading to the effects of the MSP to be biased toward the null (Nayak, 2010).

Time to resolution of diarrhea in this study was $6 \mathrm{~d}$. The duration of diarrhea was similar to some studies (Garthwaite et al., 1994; Wenge et al., 2014; Katsoulos et al., 2017); however, others have found that it to be longer (Goodell et al., 2012). Some of the differences in duration could be related to the definition of diarrhea resolution. In our study, a fecal score of $\leq 1$ defined resolution (McGuirk, 2008; Wenge et al., 2014), whereas others have used a fecal score of 0 (Goodell et al., 2012). In future studies, it is important to create a

Table 3. Results of Cox proportional hazards model evaluating days to resolution of abnormal fecal score between multispecies probiotic (MSP) and placebo (PLB) groups

\begin{tabular}{|c|c|c|c|c|c|}
\hline Variable & Description & No. & $\begin{array}{l}\text { Hazard } \\
\text { ratio }\end{array}$ & $95 \% \mathrm{CI}$ & $P$-value \\
\hline \multirow[t]{2}{*}{ Group } & PLB & 80 & Referent & & \\
\hline & MSP & 68 & 2.37 & $1.19-4.72$ & 0.01 \\
\hline \multirow[t]{4}{*}{ Time following first enrollment } & 1st quartile (0-7 d following 1st enrollment) & 36 & Referent & & \\
\hline & 2nd quartile (8-17 d following 1st enrollment) & 39 & 1.01 & $0.53-1.94$ & 0.96 \\
\hline & 3rd quartile (18-32 d following 1st enrollment) & 36 & 1.01 & $0.53-1.91$ & 0.98 \\
\hline & 4th quartile (33-81 d following 1st enrollment) & 37 & 0.76 & $0.40-1.42$ & 0.39 \\
\hline \multirow{3}{*}{$\begin{array}{l}\text { Group } \times \text { time of enrollment } \\
\text { interaction }\end{array}$} & 2nd quartile $\times$ MSP & & 0.46 & $0.18-1.18$ & 0.11 \\
\hline & 3rd quartile $\times$ MSP & & 0.33 & $0.12-0.87$ & 0.02 \\
\hline & 4th quartile $\times$ MSP & & 0.62 & $0.24-1.59$ & 0.32 \\
\hline
\end{tabular}



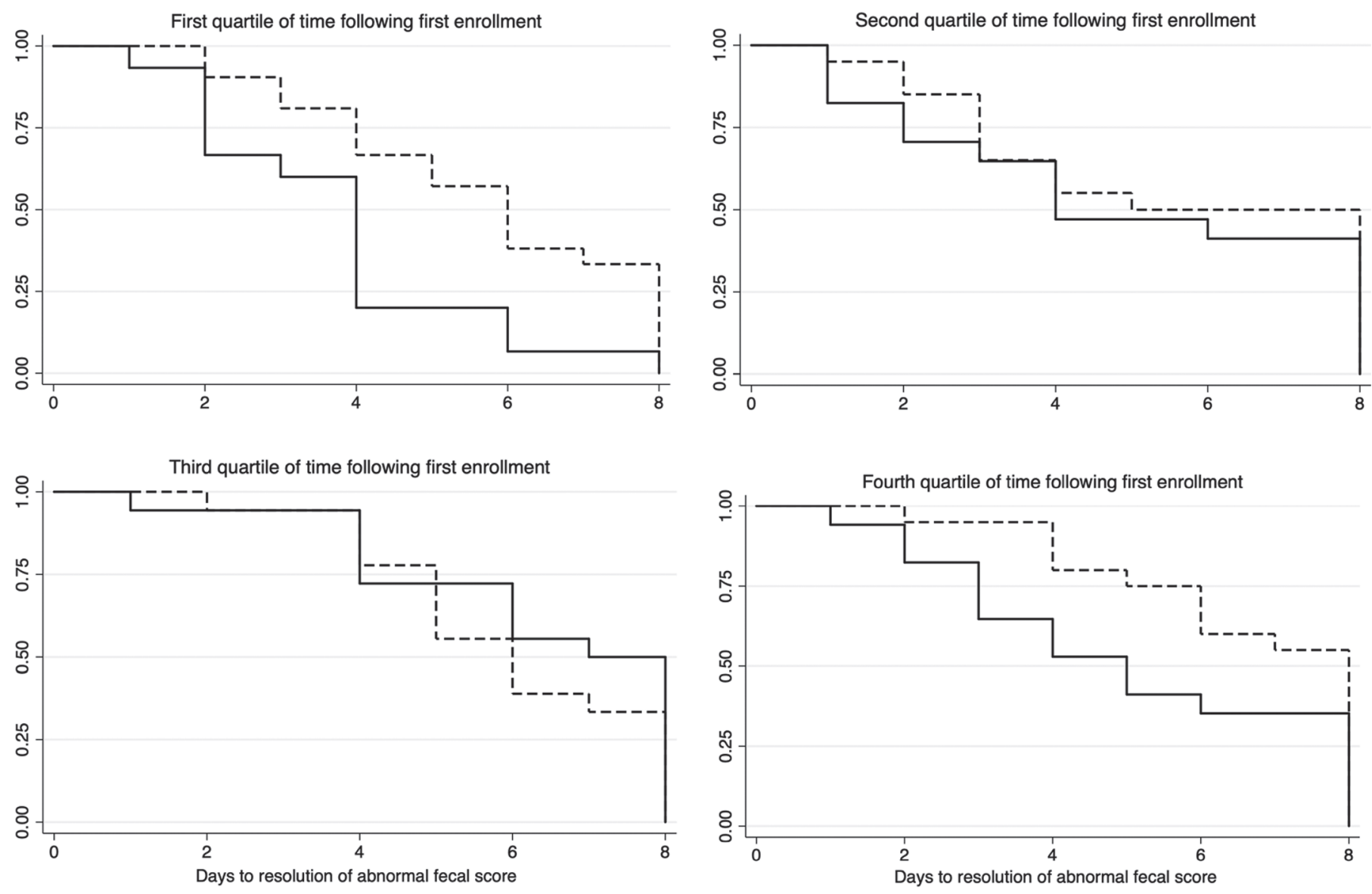

Figure 2. Results of the Kaplan Meier failure estimates evaluating days to resolution of days to an abnormal fecal score between multispecies probiotic (MSP) and placebo (PLB) groups by time from first enrollment of the experiment.

standardized definition of diarrhea resolution, taking into consideration that high volumes of milk lead to higher fecal scores (Liang et al., 2016).
The MSP contained lactic acid bacteria and Bifidobacterium, which have differing mechanisms of action in reducing or preventing diarrhea. Lactic acid bacteria

Table 4. Results of mixed multivariable linear regression model of ADG in the first week following enrollment in multispecies probiotic (MSP) and placebo (PLB) groups

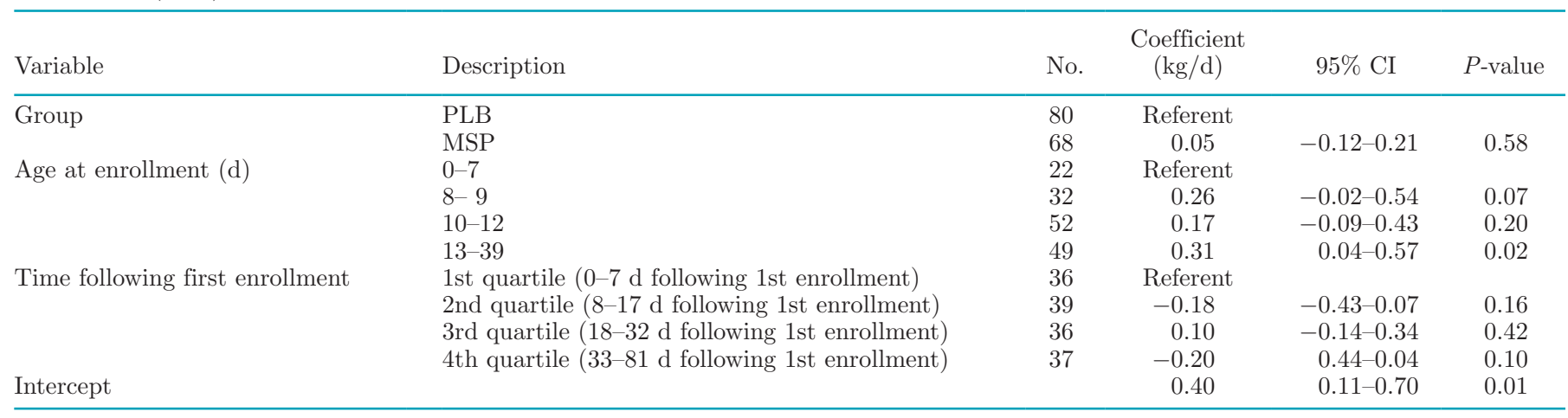


are thought to inhibit the growth of pathogenic bacteria by decreasing the $\mathrm{pH}$ in the large intestine and through competitive attachment (Riddle et al., 2010). Bifidobacterium bifidum interferes with the invasion of host epithelial cells, releases bifidocin causing cell-death in gram-positive bacteria, and synthesizes exopolysaccharides that inhibit the growth of pathogens (Sarkar and Mandal, 2016). Through these differing mechanisms, the MSP could have led to a reduced duration of diarrhea. In addition, the use of multiple strains of probiotic in our study could have been more useful than a single strain, as they can proliferate more lactic acid bacteria than a single-strain probiotic (Rolfe, 2000).

An unexpected result of the study was that the effect of the MSP was dependent on the time from enrollment of the first calf. When probiotics are stored at higher temperatures, the stability of the product is low (Kumar Anal and Singh, 2007). As the MSP was stored in nontemperature-controlled storage when left at the commercial dairy barns and was replenished in the middle of the experiment, the seasonal effect seen could be the result of high storage temperatures reducing the efficacy of MSP. Seasonal changes have also been found in regards to the pathogen load and host susceptibility that could be responsible for diarrhea, and this could have affected the efficacy of the MSP (Dowell, 2001; Huetink et al., 2001; McGuirk, 2008).

Longer periods of diarrhea will lead to an increased loss of fluids, leading to the development of dehydration, strong ion acidosis, electrolyte abnormalities, and the development of a negative energy balance (Smith, 2009). Thus, shortening the duration of diarrhea is critical; however, the relevance of the reduction seen in the MSP group is unknown. The MSP reduced the duration of diarrhea by $0.8 \mathrm{~d}$ when compared with the PLB group and could have minimized the fluid losses occurring over that time period. To gain an improved understanding of the clinical response, further studies are required to determine the effect on mortality and fluid losses when the MSP is administered to diarrheic calves.

\section{CONCLUSIONS}

The use of a multispecies probiotic and yeast bolus administered for 4 consecutive days reduced the duration of diarrhea in dairy calves; however, given the small magnitude of this reduction, further studies are required to determine the relevance of this reduction in a clinical and economic context. The probiotic bolus failed to have an effect on the growth of calves following its administration.

\section{ACKNOWLEDGMENTS}

The study was funded by Partner Animal Health (Ilderton, Ontario, Canada) and the Ontario Veterinary College (Guelph, Ontario, Canada).

\section{REFERENCES}

Allen, S. J., E. G. Martinez, G. V. Gregorio, and L. F. Dans. 2010. Probiotics for treating acute infectious diarrhoea. Cochrane Database Syst. Rev. 10:CD003048. https://doi.org/10.1002/14651858 .CD003048.pub3.

CONSORT (Consolidated Standards of Reporting Trials). 2010. CONSORT 2010 Flow Diagram. Accessed Jan. 1, 2019. http://www .consort-statement.org.

Constable, P. D. 2009. Treatment of calf diarrhea: Antimicrobial and ancillary treatments. Vet. Clin. North Am. Food Anim. Pract. 25:101-120. https://doi.org/10.1016/j.cvfa.2008.10.012.

Dohoo, I., W. Martin, and H. Stryhn. 2010. Veterinary Epidemiologic Research. VER, Charlottetown, PEI, Canada.

Dowell, S. F. 2001. Seasonal variation in host susceptibility and cycles of certain infectious diseases. Emerg. Infect. Dis. 7:369-374.

Garthwaite, B. D., J. K. Drackley, G. C. McCoy, and E. H. Jaster. 1994. Whole milk and oral rehydration solutions for calves with diarrhea of spontaneous origin. J. Dairy Sci. 77:835-843.

Goodell, G. M., J. Campbell, L. Hoejvang-Nielsen, W. Stansen, and P. D. Constable. 2012. An alkalinizing oral rehydration solution containing lecithin-coated citrus fiber is superior to a nonalkalinizing solution in treating 360 calves with naturally acquired diarrhea. J. Dairy Sci. 95:6677-6686.

Huetink, R. E. C., J. W. B. van der Giessen, J. P. T. M. Noodhuizen, and H. W. Ploeger. 2001. Epidemiology of Cryptosporidium spp. and Giardia duodenalis on a dairy farm. Vet. Parasitol. 102:53-67. https://doi.org/10.1016/S0304-4017(01)00514-3.

Katsoulos, P. D., M. A. Karatzia, C. I. Dovas, G. Filioussis, E. Papadopoulos, E. Kiossis, K. Arsenopoulos, T. Papadopoulos, C. Boscos, and H. Karatzias. 2017. Evaluation of the in-field efficacy of oregano essential oil administration on the control of neonatal diarrhea syndrome in calves. Res. Vet. Sci. 115:478-483. https:// doi.org/10.1016/j.rvsc.2017.07.029

Kumar Anal, A., and H. Singh. 2007. Recent advances in microencapsulation of probiotics for industrial applications and targeted delivery. Trends Food Sci. Technol. 18:240-251.

Liang, Y., J. A. Carroll, and M. A. Ballou. 2016. The digestive system of 1-week-old Jersey calves is well suited to digest, absorb, and incorporate protein and energy into tissue growth even when calves are fed a high plane of milk replacer. J. Dairy Sci. 99:1929-1937.

McGuirk, S. M. 2008. Disease management of dairy calves and heifers. Vet. Clin. North Am. Food Anim. Pract. 24:139-153. https://doi .org/10.1016/j.cvfa.2007.10.003.

Medrano-Galarza, C., S. J. Leblanc, A. Jones-Bitton, T. J. Devries, J. Rushen, A. M. D. Passillé, M. I. Endres, and D. B. Haley. 2018. Associations between management practices and within-pen prevalence of calf diarrhea and respiratory disease on dairy farms using automated milk feeders. J. Dairy Sci. 101:2293-2308. https: //doi.org/10.3168/jds.2017-13733.

NAHMS (National Animal Health Monitoring System). 2007. Dairy 2007: Heifer Calf Health and Management Practices on U.S Dairy Operations, 2007. US Department of Agriculture, Animal and Plant Health Inspection Service, Veterinary Services (USDA, APHIS, VS), Fort Collins, CO.

Nayak, B. K. 2010. Understanding the relevance of sample size calculation. Indian J. Ophthalmol. 58:469. https://doi.org/10.4103/0301 -4738.71673 .

Riddle, J. B., A. J. Gallegos, D. L. Harmon, and K. R. McLeod. 2010. Addition of a Bacillus based probiotic to the diet of preruminant calves: Influence on growth, health, and blood parameters. Int. J. Appl. Res. Vet. Med. 8:78-85. 
Rolfe, R. D. 2000. The role of probiotic cultures in the control of gastrointestinal health. J. Nutr. 130. https://doi.org/10.1093/jn/ 130.2.396S

Sarkar, A., and S. Mandal. 2016. Bifidobacteria -Insight into clinical outcomes and mechanisms of its probiotic action. Microbiol. Res. 192:159-171. https://doi.org/10.1016/j.micres.2016.07.001.

Signorini, M. L., L. Soto, M. Zbrun, G. Sequeira, M. Rosmini, and L. Frizzo. 2012. Impact of probiotic administration on the health and fecal microbiota of young calves: A meta-analysis of randomized controlled trials of lactic acid bacteria. Res. Vet. Sci. 93:250-258. https://doi.org/10.1016/j.rvsc.2011.05.001.

Sischo, W. M., D. W. Hird, I. A. Gardner, W. W. Utterback, K. H. Christiansen, T. E. Carpenter, C. Danaye-Elmi, and B. R. Heron. 1990. Economics of disease occurrence and prevention on California dairy farms: A report and evaluation of data collected for the national animal health monitoring system, 1986-87. Prev. Vet. Med. 8:141-156.

Smith, G. W. 2009. Treatment of calf diarrhea: Oral fluid therapy. Vet. Clin. North Am. Food Anim. Pract. 25:55-72. https://doi.org/10 1016/j.cvfa.2008.10.006

Svensson, C., K. Lundborg, U. Emanuelson, and S.-O. Olsson. 2003. Morbidity in Swedish dairy calves from birth to 90 days of age and individual calf-level risk factors for infectious diseases. Prev. Vet. Med. 58:179-197. https://doi.org/10.1016/s0167-5877(03)00046-1.
Timmerman, H. M., L. Mulder, H. Everts, D. V. Espen, E. V. D. Wal, G. Klaassen, S. Rouwers, R. Hartemink, F. Rombouts, and A. Beynen. 2005. Health and growth of veal calves fed milk replacers with or without probiotics. J. Dairy Sci. 88:2154-2165. https://doi .org/10.3168/jds.S0022-0302(05)72891-5.

Todd, C. G., S. T. Millman, D. R. Mcknight, T. F. Duffield, and K. E. Leslie. 2010. Nonsteroidal anti-inflammatory drug therapy for neonatal calf diarrhea complex: Effects on calf performance1. J. Anim. Sci. 88:2019-2028. https://doi.org/10.2527/jas.2009-2340.

Uyeno, Y., S. Shigemori, and T. Shimosato. 2015. Effects of probiotics/prebiotics on cattle health and productivity. Microbes Environ. 30:126-132. https://doi.org/10.1264/jsme2.ME14176.

Wenge, J., I. Steinhofel, C. Heinrich, M. Coenen, and L. Bachmann. 2014. Water and concentrate intake, weight gain and duration of diarrhea in young suckling calves on different diets. Livest. Sci. 159:133-140.

Windeyer, M. C., K. Leslie, S. Godden, D. Hodgins, K. Lissemore, and S. Leblanc. 2014. Factors associated with morbidity, mortality, and growth of dairy heifer calves up to 3 months of age. Prev. Vet. Med. 113:231-240. https://doi.org/10.1016/j.prevetmed.2013 .10.019. 